\title{
The rare cause of recurrent fever in adults: PFAPA syndrome
}

\author{
Abdurrahman Kaya ${ }^{*}$, Sibel Yıldız Kaya ${ }^{1}$, Mutlu Karkucak ${ }^{2}$, Mustafa Alkan ${ }^{1}$ and Yusuf Emre Özdemir ${ }^{1}$ \\ ${ }^{1}$ Department of Infectious Diseases, Cerrahpasa, Istanbul University, Turkey \\ ${ }^{2}$ Associate professor, Department of medical genetics, Cerrahpasa, Istanbul University, Turkey
}

\begin{abstract}
The Periodic fever with aphthous stomatitis, pharyngitis and adenitis (PFAPA) is a periodic fever syndrome. It is generally seen in children but very rarely observed in adults. The etiology of PFAPA is unknown. its attacks mostly stop before 10 years of age. Therefore, its prognosis is favorable and it has been rarely reported in adults. The diagnosis of PFAPA is merely based on clinical grounds. Many drugs such as NSAID and glucocorticoids have been given in controlling symptoms of the disease. Here, we reported a patient with PFAPA syndrome who is rarely encountered in adults.
\end{abstract}

\section{Introduction}

The PFAPA is a sporadic syndrome that is characterized by Periodic Fever with Aphthous stomatitis, Pharyngitis and Adenitis. It is generally seen in children but very rarely observed in adults. The first cases were reported in adults in 2008 [1]. Although many theories have been considered, the etiology of PFAPA is unknown. The main treatment options for the disease are unclear. But many drugs such as NSAID and glucocorticoids have been given in controlling symptoms of the disease. Here, we reported a patient with PFAPA syndrome who is rarely encountered in adults.

\section{Case}

34-year-old patient admitted to our department with fever, aphthous stomatitis, and throat pain. On physical examination, pharyngitis without tonsillar exudates, aphthous and painful ulcers in the mouth and bilateral multiple lymphadenopathies in cervical region were present. In the medical history, these symptoms had started first after his tooth was pulled out 45 days ago. The symptoms had regressed within 7 days after using antibiotics combined with analgesic. But, it had recurred after around 30 days. It had been thought that they might be related to the filled tooth and then other tooth had been pulled out. He visited many doctors who is specialized in different field and took many antibiotics such as fluoroquinolones, beta lactams and macrolides. But he had not recovered, and the attack had repeatedly recurred. The history of periodic fever in his family, especially FMF was absent. Laboratory as follow: White blood cell: $12000 \mathrm{C}$ - reactive protein: $75 \mathrm{mg} / \mathrm{L}$, sedimentation rate: 55/hour. Imaging studies, including panoramic dental X-ray, chest films, sinus film were normal. Abscess and mass were not detected on ultrasound and computer tomography except for bilateral cervical LAP. Viral serologies were unremarkable. MEFV (FMF gene) mutation analysis and rose Bengal test were negative. Throat and blood cultures were negative. Antinuclear antibody and procalsitonin are within normal ranges. Acute phase reactants were elevated during episodes but normalize between attacks. The patient was well between the episodes. After searching for in literature, the patient was considered as PFAPA syndrome because he met both diagnostic criteria and exclusion criteria of the PFAPA syndrome. Prednisolone $60 \mathrm{mg} / \mathrm{kg}$ was given at the first days of the next attack. Subsequently, the patient recovered dramatically within hours. On follow-ups for two years, prednisolone was just given at the first days of attacks and the syndrome has been recurred six weeks intervals (Figure 1).

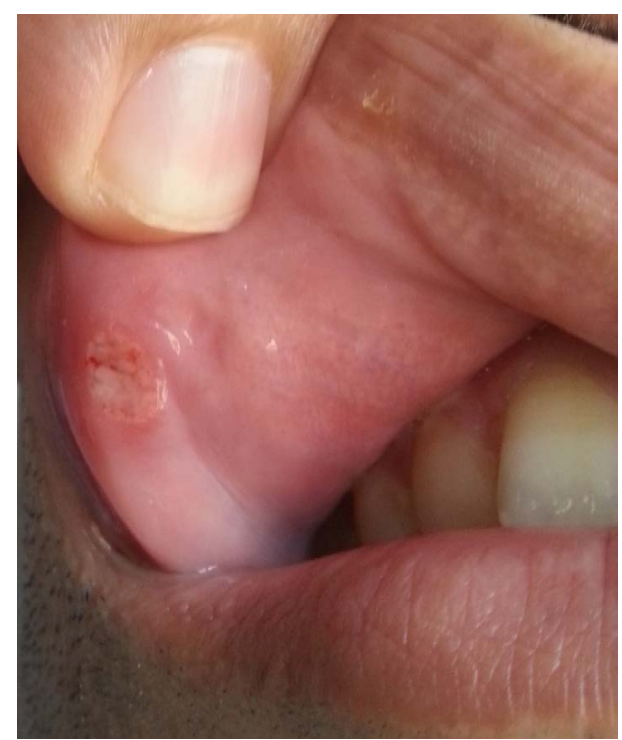

Figure 1. A round, yellow-grey and painful aphthous ulcer on the upper lip

*Correspondence to: Abdurrahman Kaya, Department of Infectious Disease, Suleymaniye Gynecology, Obstetrics and Children disease Training and Research Hospital, İstanbul, Turkey, Tel: 905066113328, Fax: 902126320050, E-mail: dr.abdkaya@hotmail.com

Key words: PFAPA syndrome, periodic fever, aphthous stomatitis

Received: August 06, 2018; Accepted: September 17, 2018; Published: September 22, 2018 


\section{Discussion}

PFAPA syndrome is a self-limited disease. Its attacks mostly stop before 10 years of age. Therefore, its prognosis is favorable, and it has been rarely reported in adults. The syndrome is male predominance and the patient with familial cases are rarely observed [2-6]. In this patient, there was no history of familial cases. Fever is the main symptom of the syndrome that starts abruptly and often falls to normal in a week. Fever occurred at similar intervals and chills and malaise may be accompanied by fever. As periodic fever syndrome, PFAPA is similar to FMF which is endemic in our country but have not regular attacks. The attacks of the patient were regularly, and the patient did not have abdominal pain. Furthermore, FMF does not also respond to glucocorticoids treatment. In this syndrome, aphthous ulcers were observed about $40 \%-70 \%$ of the patient, mostly on the lips (picture) or buccal mucosa [2]. It is seen a few, small, easily missed, yellow-grey and painful aphthae. Because of the recurrent aphthous ulcers, it resembles Behçet disease which is seen in our country. But adenitis and pharyngitis are not observed in Behçet disease, not presenting periodic fever disease as well, and he does not have genital ulcers and uveitis. Laboratory findings usually showed moderate leukocytosis, mild elevated acute phase reactants during attacks. They are generally within normal ranges between episodes. The diagnosis of PFAPA is merely based on clinical grounds. Our case met the diagnostic criteria for PFAPA [7]. It may be confused with tonsillitis or a viral syndrome, the diagnosis can be delayed or missed in adults since such patients are rarely encounter in clinical practice. Also, it is difficult to recognize the fixed periodicity. We initially thought that he responded to antibiotics and NSAID which is one of its therapies. But these therapies delayed the diagnosis of the disease in this case. It is important to early diagnose PFAPA since it causes to perform unnecessary and expensive laboratory tests and cause to take potentially harmful treatments.

As a conclusion, as in this case, it is very challenge to diagnose the syndrome in adults and us infectious disease specialists do not practically encounter such patients in adults. PFAPA syndrome should be kept in mind in adults if the patient has periodic fever and the symptoms do not respond to the medical treatment.

\section{References}

1. Shai Padeh M, Stoffman N, Berkun Y (2008) Periodic fever accompanied by aphthous stomatitis, pharyngitis and cervical adenitis syndrome (PFAPA syndrome) in adults. Isr Med Assoc J 10: 358-60. [Crossref]

2. Shai Padeh M (2014) periodic fever with aphthous stomatitis, pharyngitis and adenitis (PFAPA syndrome).

3. Adachi M, Watanabe A, Nishiyama A, Oyazato Y, Kamioka I, et al. (2011) Familial cases of periodic fever with aphthous stomatitis, pharyngitis, and cervical adenitis syndrome. Pediatrics 158: 155-159. [Crossref]

4. Antón-Martín P, Movilla RO, Martín SG, Allende LM, Rubio MTC, et al. (2011) PFAPA syndrome in siblings. Is there a genetic background? Eur J Pediatr 170: 15631568. [Crossref]

5. Sampaio ICRM, Rodrigo MJ, Marques JGDPM (2009) Two siblings with periodic fever, aphthous stomatitis, pharyngitis, adenitis (PFAPA) syndrome. Pediatr Infect Dis J 28: 254-255. [Crossref]

6. Valenzuela PM, Majerson D, Tapia JL, Talesnik E (2009) Syndrome of periodic fever, aphthous stomatitis, pharyngitis, and adenitis (PFAPA) in siblings. Clin Rheumatol 28: 1235-1237. [Crossref]

7. Padeh S (2005) Periodic fever syndromes. Pediatr clin of N Am 52: 577-609.

Copyright: (C2018 Kaya A. This is an open-access article distributed under the terms of the Creative Commons Attribution License, which permits unrestricted use, distribution, and reproduction in any medium, provided the original author and source are credited. 